\title{
Epigenetic approaches in the treatment of myelodysplastic syndromes: clinical utility of azacitidine
}

This article was published in the following Dove Press journal:

OncoTargets and Therapy

21 July 2010

Number of times this article has been viewed

\section{Steven E McCormack Erica D Warlick}

Department of Medicine, Division of Hematology, Oncology, and Transplantation, University of Minnesota, Minneapolis, Minnesota, USA
Correspondence: Erica D Warlick Blood and Marrow Transplant Program, University of Minnesota, Mayo Medical Code 480, 420 Delaware St SE, Minneapolis, MN 55455, USA

$\mathrm{Tel}+\mathrm{I}$ 6/2-625-5467

$\mathrm{Fax}+$ I 612-625-6919

Email ewarlick@umn.edu
Abstract: Myelodysplastic syndromes (MDS) are a varied group of diseases leading to significant morbidity and mortality. Therapy of MDS has been difficult, with supportive cares used to ameliorate symptoms, and hematopoietic stem cell transplantation the only curative option. Agents, such as the cytidine analog azacitidine, exert an effect on DNA methyltransferase leading to a reduction in DNA methylation, a process thought to be key to the pathogenesis of MDS. Recently, azacitidine has been shown to prolong survival and improve quality of life in patients with MDS, while maintaining a favorable adverse effect profile. This review highlights the scientific rationale for the use of azacitidine in addition to its application in current clinical practice for patients with MDS.

Keywords: hypomethylation, epigenetics, myelodysplastic syndromes, azacitidine

\section{Introduction}

Myelodysplastic syndromes (MDS) are a complex and heterogeneous group of clonal stem cell disorders manifested by diverse clinical and biologic paths with varying need for transfusions, risk of infection, and risk of progression to acute leukemia. The US Food and Drug Administration (FDA) approval of 3 new therapeutic agents for MDS over the last few years (azacitidine, lenalidomide, and decitabine), as well as innumerable available clinical trials, has changed the treatment paradigm for this spectrum of diseases; however, stem cell transplantation remains the only curative therapy for MDS.

MDS pathophysiology is complex, diverse, and still not completely understood. Structural alterations in DNA, as evident by the cytogenetic abnormalities seen in a majority of patients, play a role in the pathogenesis of disease due to loss or alteration of genetic material involved in proliferation, differentiation, or apoptosis. Epigenetic changes in the form of modifications to the transcriptional capacity of the cell via processes, such as DNA methylation or histone acetylation, can also alter gene expression impacting disease biology. ${ }^{1}$ As such, DNA hypermethylation of key cellular machinery involved in cell cycle regulation, apoptosis, and tumor suppressor control is well documented in the pathogenesis of MDS, as well as in other cancers. The tumor suppressor and cell cycle regulatory gene $\mathrm{p} 15^{\mathrm{INK} 4 \mathrm{~B}}$ is an example of one important gene noted to be hypermethylated in MDS resulting in uncontrolled cell cycle progression and cellular proliferation. ${ }^{1-6}$

Hypermethylated DNA sequences of key cellular machinery provide a biologically rational therapeutic target for MDS. Currently, both FDA-approved hypomethylating agents (azacitidine and decitabine) have shown good clinical responses in patients 
with MDS with recent data confirming an overall survival (OS) benefit with azacitidine therapy. ${ }^{7-11}$ This review will highlight azacitidine pharmacology, explore azacitidine efficacy studies both as single-agent therapy and in combination with other drugs, compare available data between azacitidine and decitabine, address the impact on patient quality of life (QOL), and discuss the role of azacitidine in the comprehensive care of patients with MDS.

\section{Pharmacology Mechanism of action}

Azacitidine (5-azacytidine; Vidaza; Celgene Corporation, Summit, NJ, USA) was the first drug approved for the treatment of MDS by the FDA. ${ }^{12}$ Azacitidine is an analog of cytidine, a pyrimidine nucleoside that is a component of human RNA. Although azacitidine falls into the category of hypomethylating agents, its mechanism of action is likely multifactorial.

The effectiveness of azacitidine is contingent on its entry into cells via nucleoside transporter proteins. The specific protein responsible for azacitidine transport remains unclear. ${ }^{13}$ After multiple phosphorylation steps, azacitidine is largely $(\sim 80 \%)$ incorporated into RNA, where it potentially exerts a cytotoxic effect by inhibiting protein translation. ${ }^{13,14}$ Whether or not azacitidine plays a role in RNA hypomethylation has yet to be determined. ${ }^{14}$ Ribonucleotide reductase converts a portion $(\sim 10 \%-20 \%)$ of phosphorylated azacitidine to 5-aza-dCDP, a phosphorylated metabolite of 5-aza-2'-deoxycytidine (decitabine; Dacogen; Eisai Incorporated, Woodcliff Lake, NJ, USA). The compound 5-aza-dCDP is further phosphorylated and incorporated into DNA as azacytosine. ${ }^{14}$

In the environment of malignancy, DNA methyltransferase (DNMT) has been known to hypermethylate cytosine residues at cytosine-guanine repeat sequences $(\mathrm{CpG}$ islands). ${ }^{15}$ The incorporation of azacytosine into DNA leads to inhibition of DNMT. ${ }^{16}$ DNMT recognizes the modified azacytosine-guanine regions as native $\mathrm{CpG}$ islands and in turn forms a covalent complex with the abnormal dinucleotide regions. ${ }^{13}$ It is believed that DNMT becomes "trapped", which eventually leads to its degradation and subsequent inability to hypermethylate $\mathrm{CpG}$ islands. ${ }^{17}$ Through this process, subsequent DNA copies are able to propagate without the potential harmful effects of hypermethylation. ${ }^{15}$ By reducing the quantity of hypermethylated DNA produced, azacitidine can serve to limit the proliferation of malignant cells, while allowing an increased proportion of normal cells to differentiate. Interestingly, methylation reversal has not been shown to consistently correlate with clinical response highlighting our incomplete understanding of the full spectrum of hypomethylating agent activity. ${ }^{18,19}$ Although there is some suggestion that DNA methylation may be predictive of outcomes for patients with MDS, currently there is no standard role for clinically monitoring methylation in patients treated with hypomethylating agents. ${ }^{20}$

In the 1960s-1980s, azacitidine was studied in a variety of malignancies, focusing primarily on hematologic malignancies. When used at high doses $\left(150-400 \mathrm{mg} / \mathrm{m}^{2}\right)$, in initial studies, azacitidine predominantly exhibited direct cytotoxicity. Unfortunately, in that manner, its use was limited by significant toxicities and was only variably effective. ${ }^{21-24}$ With greater mechanistic understanding of azacitidine's potential hypomethylating effects, the variable efficacy with high-dose therapy is understandable given the need for cellular survival to produce hypomethylated progenitor DNA.

\section{Dosing}

The FDA-approved dosing scheme for azacitidine is outlined in the FDA approval summary. ${ }^{25}$ Azacitidine is approved for patients with MDS. The starting dose has been recommended at $75 \mathrm{mg} / \mathrm{m}^{2}$ administered either subcutaneously (SQ) or intravenously (IV) for 7 straight days out of a 28-day cycle. The dose can be increased to $100 \mathrm{mg} / \mathrm{m}^{2}$ if there is no effect and no dose-limiting toxicity after 2 cycles. Administration of azacitidine is recommended to continue as long as there is a clinical benefit in the absence of dose-limiting toxicities. There are no first-cycle dose adjustments currently recommended regardless of the patient's peripheral blood counts or renal function given the nature of the cytopenias inherent in MDS. Adjustments may be made for subsequent cycles based on hematologic status, alteration in renal function, or change in serum bicarbonate levels. It is important to note that azacitidine is contraindicated in patients with diffuse hepatic metastases. ${ }^{25}$

A recent trial suggested that SQ azacitidine administered over 5 consecutive days $\left(75 \mathrm{mg} / \mathrm{m}^{2} / \mathrm{d}\right), 5$ consecutive days with 2 days off followed by 2 more days of administration $\left(75 \mathrm{mg} / \mathrm{m}^{2} / \mathrm{d}\right)$, or 5 consecutive days with 2 days off followed by 5 more days of administration $\left(50 \mathrm{mg} / \mathrm{m}^{2} / \mathrm{d}\right)$ may be reasonable dosing alternatives for those patients in the lower risk MDS International Prognostic Scoring System (IPSS) categories of low/INT-1 (intermediate-1) with reported responses demonstrating transfusion independence and hematologic improvement (HI). ${ }^{26}$ These alternative dosing schedules are not FDA-approved but may offer a greater convenience to the patient providing a potentially similar 
benefit to the established dosing regimen in patients whose treatment goals are palliative. Studies evaluating the safety and efficacy of oral azacitidine are underway and may offer even greater convenience to patients in the future..$^{27,28}$

\section{Pharmacokinetics}

Although azacitidine was initially studied with IV delivery, it is also approved for SQ injection. Azacitidine has a high bioavailability after SQ injection (up to $89 \%$ of the equivalent IV dose). Peak plasma concentration of azacitidine is reached 30 minutes after SQ injection, and the plasma half-life of SQ azacitidine is 41 minutes. Azacitidine has a large volume of distribution, with an apparent preferential uptake in tumor tissues although it is unclear how successfully azacitidine can cross the blood-brain barrier. ${ }^{29,30}$ There are data to suggest that 5-aza-2'-deoxycytidine (decitabine) can reach measurable CSF concentrations in animal models. ${ }^{31}$ Excretion appears to be largely kidney dependent (50\%-90\%).

\section{Efficacy and safety}

\section{Azacitidine as a single agent for MDS}

The use of azacitidine was initially investigated in a wide variety of clinical scenarios and at generally higher doses than currently approved. ${ }^{21-24}$ In order to limit toxicities and take advantage of the recognized mechanism of DNA hypomethylation, azacitidine was further investigated at significantly lower doses and primarily in hematologic malignancies.

Silverman and colleagues ${ }^{32}$ led both laboratory- and patient-based investigations into low-dose azacitidine for therapy for MDS. Two phase 2 studies through the Cancer and Leukemia Group B (CALGB) evaluated azacitidine given at $75 \mathrm{mg} / \mathrm{m}^{2}$ IV (CALGB 8421) or SQ (CALGB 8921) for 7 consecutive days on a 28-day cycle. Based on updated IWG (International Working Group) 2000 response criteria, patients receiving IV azacitidine (8421) or SQ azacitidine (8921) had responses (combined rates of complete, partial, or improved responses) of $44 \%$ and $40 \%$, respectively. ${ }^{10,33}$

The data earning azacitidine its FDA approval was published in 2002 by Silverman and colleagues ${ }^{9}$ and updated in 2006 with new IWG 2000 response criteria. The CALGB 9221 study was a phase 3 randomized control trial of low-dose SQ azacitidine for patients with MDS. This multi-institutional trial included 191 patients randomized to either therapy with azacitidine ( $75 \mathrm{mg} / \mathrm{m}^{2}$ for days $1-7$ of a 28 -day cycle) or supportive care (transfusions as needed, no hematopoietic growth factors). Azacitidine could be increased to $100 \mathrm{mg} / \mathrm{m}^{2}$ after 2 full cycles if there was no improvement and there were no dose-limiting toxicities. The 2 groups were comparable in terms of FAB classifications, IPSS scores, time from diagnosis to study entry, and cytogenetic groupings. In retrospect, a central pathology review of marrow specimens determined that 19 patients should be classified as acute myeloid leukemia (AML) although this did not ultimately affect the results. These patients were excluded from an analysis of progression to AML. Importantly, patients in the supportive care arm were allowed to cross over to the azacitidine arm after at least 4 months of supportive care or if they met other criteria for worsening disease status prior to that time. ${ }^{9}$

With outcome data based on the IWG 2000 response criteria, of 99 patients in the azacitidine arm, 10\% obtained complete remission (CR), 1\% partial remission (PR), and 36\% with $\mathrm{HI}$ for a total overall response rate (ORR) of $47 \% .{ }^{10}$ The supportive care arm (prior to any crossover) showed an ORR of $17 \%$ with all responses manifest as $\mathrm{HI} .{ }^{9}$ Fifty-one patients crossed over from supportive care to azacitidine and obtained a CR rate of $6 \%$, PR of $4 \%$, and $\mathrm{HI}$ of $25 \%$ for an ORR of $35 \% .{ }^{10}$ Of the 65 patients receiving azacitidine who were red blood cell (RBC) transfusion-dependent at the start of the trial, $45 \%$ became transfusion-independent. ${ }^{9}$ The investigators noted that most patients who responded did not demonstrate this response until 3 or 4 cycles of therapy. ${ }^{9}$

Within CALGB 9221, treatment with azacitidine was shown to prolong time to leukemic transformation or death (treatment failure). The mean time to treatment failure for patients receiving azacitidine and supportive care was 21 months and 12 months, respectively $(P=0.007)$. There was a trend toward improved OS in those treated with azacitidine, with a median of 20 months compared with 14 months for patients in the supportive care arm. Although this finding did not reach statistical significance $(P=0.10)$ possibly due to the effect of patient crossover, a subset survival analysis of patients receiving azacitidine compared with patients who either never crossed over or crossed over after 6 months of supportive care did reveal a statistically significant survival advantage highlighting the impact of azacitidine on the natural history of disease and importance of use earlier in the patient's disease course. ${ }^{21}$ Silverman et $\mathrm{al}^{9}$ concluded that the use of azacitidine improved time to progression or death, reduced the rate of RBC transfusions, and improved measurable hematologic parameters when compared with best supportive care (BSC).

The CALGB 9221 trial also evaluated QOL outcomes. ${ }^{34}$ Based on QOL questionnaires and telephone surveys, patients in the azacitidine group had significantly decreased fatigue and dyspnea with improved physical functioning and positive affect when compared with patients in the supportive 
care arm. Patients who crossed over to azacitidine therapy had significant improvements in the same areas. The effects seemed most pronounced in patients who received at least 4 cycles of azacitidine, which did correlate to the objective HIs seen in the outcomes data outlined above. The authors felt that these QOL improvements were valid and not explained by other effects. ${ }^{34}$

Based on the promising outcomes from the CALGB azacitidine studies, the International Vidaza High-Risk MDS Survival Study Group developed AZA-001, a multicenter phase 3 randomized controlled trial for patients with MDS with higher IPSS risk score (intermediate-2/high) randomizing patients to either azacitidine vs one of 3 conventional care regimens (BSC, low-dose cytarabine, or intensive chemotherapy). ${ }^{11}$ Prior to randomization, investigators preselected which conventional care regimen would be the most appropriate for a given patient if they were randomized to the conventional care arm. They were then randomized either to receive SQ azacitidine $\left(75 \mathrm{mg} / \mathrm{m}^{2}\right.$ for days $1-7$ of a 28 -day cycle) or to their designated conventional care regimen. No crossover was allowed in this study, and patients could not receive erythropoietin (EPO) analogs. Patients with treatment-related MDS were excluded. Patients also had to be of good performance status and could not have been previously treated with azacitidine or have an upcoming planned allogeneic hematopoietic stem cell transplant (HCT). ${ }^{11}$

Of 358 patients, 179 patients were randomized to receive azacitidine. For the 179 patients receiving conventional care, 105 received $\mathrm{BSC}, 49$ received low-dose cytarabine, and 25 received intensive chemotherapy. The outcome analysis was based on an intention to treat. Central review highlighted a few notable deviations: (1) Eighteen patients should have been assigned an IPSS score of INT-1 (5 in the azacitidine group and 13 in the conventional care group), (2) One-hundred and thirteen of the 358 patients $(32 \%)$ could be diagnosed as AML based on current World Health Organization criteria, and 3) Eight patients (4 who received azacitidine) went on to receive an unplanned allogeneic HCT. ${ }^{11}$ Of 358 patients, 4 in the azacitidine group and 13 in the conventional care group never received therapy but were included in the intention-to-treat analysis. For those patients treated with azacitidine, the median number of cycles given was 9 , and $86 \%$ of those patients required no azacitidine dose adjustments.

The study's primary end point was OS. Median OS was significantly improved (24.5 months vs 15 months) for patients receiving azacitidine $(P=0.0001)$. The Kaplan-Meier estimate for 2-year survival for the azacitidine patients was significantly improved (50.8\% vs $26.2 \%)$ over the conventional care regimens $(P<0.0001)$. This OS benefit persisted on analysis of cytogenetic subgroups, including patients with abnormalities of chromosome $7 .{ }^{11}$ OS subgroup analysis based on the initial potential conventional care arm assigned (BSC only vs low-dose cytarabine vs intensive chemotherapy) retained a statistically significant survival benefit in the BSC and low-dose cytarabine groups. However, for patients initially potentially designated for intensive chemotherapy if randomized to the conventional care arm, the use of azacitidine resulted in an improved median OS of 25.1 months vs 15.7 months for those patients who did receive intensive that did not reach statistical significance. $(P=0.51)$. The lack of statistical significance is likely due to the relatively low number of patients in this designated group (total of 42). ${ }^{23}$

In the entire cohort of patients, the time to transformation to AML (defined in the study as bone marrow blast percentage of $>30 \%$ ) was delayed to 17.8 months for those receiving azacitidine vs 11.5 months for those in the conventional care arm $(P<0.00001)$. Subgroup analysis, based on the original potential preselected conventional care arm, revealed a significant improvement in time to leukemia progression in the azacitidine vs BSC arm (15 months vs 10.1 months). However, the trend didn't reach significance in those patients treated with azacitidine vs low-dose cytarabine or intensive chemotherapy. ${ }^{11}$

AZA-001 trial was the first to prospectively confirm azacitidine's impact on OS in high-risk de novo patients with MDS. Subsequent meta-analysis reviewing 4 large hypomethylation MDS trials confirmed the OS benefit with azacitidine therapy. ${ }^{35}$ Future study including patients with treatment-related MDS are warranted to determine if this survival benefit also applies to that patient population.

\section{Azacitidine in combination with other agents}

Currently, there are no FDA-approved regimens that include azacitidine in combination with other drugs for therapy of MDS. Several studies have evaluated azacitidine in combination with other cytotoxic agents or with other epigenetic-derived therapy, such as histone deacetylase inhibitors. A large, multicenter, phase 3 ECOG study evaluating the combination of azacitidine with MS-275, a histone deacetylase inhibitor, is currently underway based on promising phase 1 combination data. Azacitidine in combination with sodium phenylbutyrate, with valproic acid alone, with all-trans retinoic acid, hydroxyurea, and gemtuzumab ozogamicin, and with thalidomide has also been evaluated in early-phase studies. ${ }^{36-40}$ The majority of these studies have included patients with either 
high-risk MDS or AML progressing on standard therapies. Generally, the combinations have been deemed safe and have shown some clinical responses. ${ }^{36-40}$

Recent phase 1 data using the combination of azacitidine and lenalidomide in patients with high-risk MDS has shown promising results. Six dosing schedules were assessed with a maximum tolerated dose not found. The combination was found to be safe and tolerable, and the CR rate of $44 \%$ is slightly higher than outcomes of each individual singledrug study alone. Future phase 2 testing is underway with a dosing schedule of azacitidine $75 \mathrm{mg} / \mathrm{m}^{2}$ on days $1-5$ and lenalidomide $10 \mathrm{mg}$ daily on days $1-21$ to determine if the combination provides improved responses compared with either azacitidine or lenalidomide alone. ${ }^{41}$

\section{Azacitidine prior to HCT}

Randomized trials evaluating the safety or efficacy of azacitidine administration prior to HCT are currently lacking. Use of both azacitidine and decitabine prior to allogeneic stem cell transplantation with either sibling or matched unrelated donors have been reported without increasing transplantrelated toxicity. ${ }^{42,43}$ However, the impact of pretransplant hypomethylating therapy on posttransplant outcomes, such as incidence of relapse, OS, and disease-free survival, is unknown. Recent data indicate that azacitidine may have an immune-modulating effect on $\mathrm{T}$ cells, with potential implications on graft-vs-host disease in the transplant setting; thus, further investigation is warranted. ${ }^{44}$

\section{Safety and tolerability}

In early trials, higher IV doses $\left(100-400 \mathrm{mg} / \mathrm{m}^{2}\right)$ of azacitidine were associated with significant nausea, vomiting, infusion reactions, in addition to severe hematologic toxicities. ${ }^{21-24}$ Additionally, in previous studies evaluating azacitidine in patients with metastatic tumors or with concurrent cirrhosis, severe hepatic toxicity was seen. As a result, azacitidine is contraindicated in patients with significant hepatic involvement by malignancy. ${ }^{12,30}$

With current dosing schemes, azacitidine is generally well-tolerated. Interestingly, a stringent evaluation of adverse effects is made difficult by similar adverse events reported in both patients receiving azacitidine and those patients in supportive care arms who suffered from sequelae due to the natural history of their MDS. ${ }^{30}$ The documented adverse effects are primarily hematologic. Patients receiving azacitidine on the AZA-001 trial frequently experienced grade 3 or 4 neutropenia $(91 \%)$, thrombocytopenia $(85 \%)$, or anemia $(57 \%)$ during their course of care compared with the patients in conventional care arms at $76 \%, 80 \%$, and $68 \%$, respectively. ${ }^{11}$ Given the inherent biology of MDS, attribution of cytopenias to azacitidine vs MDS itself is challenging. Patients treated with azacitidine generally do have an increase in transfusion requirements during their first cycles of therapy, although this effect disappears in those with a positive response to therapy. ${ }^{9}$ In addition, the important effect noted in the AZA-001 trial was that azacitidine therapy did not result in an increased risk of infection. ${ }^{11}$ For patients who discontinued therapy early due to adverse effects, the reason was generally due to hematologic toxicities. ${ }^{10,11,30}$ Additionally, in CALGB studies, there was no increased risk of bleeding events in patients on azacitidine. ${ }^{10}$ In summary, cytopenias inherent to both therapy and MDS did not lead to increased risk of bleeding or infection in those patients treated with azacitidine, and for those who responded to therapy, resolution of cytopenias occurred with a median time to response of 3 months.

Certain nonhematologic toxicities commonly documented in patients receiving azacitidine included nausea, vomiting, constipation, diarrhea, anorexia, fatigue, arthralgias, headache, hepatic function abnormalities, and injection site reactions (with anecdotal reports suggesting primrose oil to ameliorate the SQ injection site reactions). ${ }^{10,11,30,45}$ Also, adverse effects are more frequently reported within the initial 2 cycles of therapy. ${ }^{30}$ Although nausea and vomiting were a dose-limiting toxicity in the 1960s-1980s, presumably modern-day antiemetic therapy has played a role in improving the tolerability of azacitidine.

\section{Patient perspectives in MDS and azacitidine}

Problems stemming from the ineffective hematopoiesis hallmark to MDS greatly impacts patient's QOL. Due to the subjective impact of disease burden on QOL, health-related quality-of-life assessment tools have been developed to objectively evaluate the impact of disease and treatment on patient's overall life satisfaction. Numerous tools exist with the Functional Assessment of Cancer Therapy (FACT) based evaluations and the European Organization for Research and Treatment of Cancer (EORTC) Core Quality of Life Questionnaire (QLQ-C30), the tools most frequently cited. Although an exhaustive review on QOL in MDS is beyond the scope of this review, a recent publication by Pinchon et $\mathrm{al}^{46}$ summarizes the available QOL data based on impact of $\mathrm{RBC}$ transfusions and symptoms associated with anemia on patients with MDS. Most notable, the findings suggest that generally patients whose hemoglobin level rises and transfusion requirements decrease have improved QOL. These results were evident in those treated solely with growth 
factor support (EPO and darbepoetin alfa with or without granulocyte-colony-stimulating factor), as well as in those treated with hypomethylating agents. ${ }^{7,34,47-52}$ Other QOL tools have identified the important impact of fatigue on daily function and ability to work or do desired activities. Additional factors, such as fever, infections, weight loss, and bleeding, impacted QOL to a lesser degree. ${ }^{53}$ These data clearly demonstrate the dramatic impact of MDS-related complications on patient's QOL.

Based on azacitidine's efficacy via improved blood counts, transfusion independence, remissions, prolonged OS, and delayed time to leukemic transformation, a positive impact on QOL is expected. QOL assessments were a key component to the outcomes assessed in CALGB 9221 randomizing patients to azacitidine vs BSC and confirmed this impact. Specifically, patients in the azacitidine arm noted significant improvements in fatigue, physical function, dyspnea, psychosocial distress, and affect. ., $^{94}$

Although azacitidine is filled with promise of potential clinical benefits, the therapy is not without possible complications and frustrations. As with any chemotherapy, initial treatment is associated with worsening of baseline cytopenias and an expected increased rate of transfusions until a response is manifest. However, based on the mechanism of action and expected slower onset of response, patience and perseverance to continue therapy are required for sometimes a prolonged period of time before seeing a clinical benefit. With a median time to response of 3 cycles translating into $80+$ days, adherence to therapy by both patient and physician for a minimum of 4 cycles is crucial to allow enough time to until response assessment. $^{10}$

\section{Additional therapeutic options in MDS Decitabine}

Decitabine (5-aza-2'-deoxycytidine) is a cytosine analog that, through a series of metabolic steps differing from azacitidine, eventually incorporates into DNA. ${ }^{14}$ At low doses, decitabine can lead to DNMT degradation and subsequent hypomethylation, whereas higher doses lead to direct cytotoxicity. ${ }^{14}$ Initial studies using a dosing schedule of $15 \mathrm{mg} / \mathrm{m}^{2}$ IV over 3 hours every 8 hours for a total of 3 days repeated every 6 weeks yielded an ORR of $17 \%$ with a CR rate of $9 \%{ }^{7}$ Subsequent studies using alternative dosing regimens more appropriate for outpatient use $\left(20 \mathrm{mg} / \mathrm{m}^{2}\right.$ days $1-5 \mathrm{SQ}, 20 \mathrm{mg} / \mathrm{m}^{2}$ days 1-5 IV, and $10 \mathrm{mg} / \mathrm{m}^{2} \mathrm{IV}$ days $1-10$ ) produced higher CR rates and ORRs. The dosing schedule of $20 \mathrm{mg} / \mathrm{m}^{2} \mathrm{IV}$ days $1-5$ yielded the highest CR rates (39\%) along with evidence of $\mathrm{p} 15^{\text {Ink4B }}$ hypomethylation in those achieving a CR. ${ }^{8}$ Given these good responses, the above dosing schedule is the one most frequently used in practice.

Recent meta-analysis of 4 significant azacitidine and decitabine trials confirmed that hypomethylating agents improve survival compared with BSC (hazard ratio $[\mathrm{HR}]=0.66 ; 95 \%$ confidence interval $[\mathrm{CI}], 0.55-0.8)$, prolong time to AML transformation or death $(\mathrm{HR}=0.69$; 95\% CI, 0.58-0.82), and improved CR (HR = 7.63; 95\% CI, 1.41-41.17), PR ( $\mathrm{HR}=6.01 ; 95 \% \mathrm{CI}, 2.93-12.31)$, and $\mathrm{HI}$ rates $(\mathrm{HR}=3.06 ; 95 \% \mathrm{CI}, 1.09-8.6)$. Interestingly, freedom from RBC transfusions was not improved with hypomethylating agents compared with conventional care $(\mathrm{HR}=10.65 ; 95 \%$ CI, 0.29-388.92). Hypomethylating agents were, as expected from individual trial data, associated with cytopenias and associated febrile neutropenia and a higher treatment-related mortality compared with conventional care. ${ }^{35}$

Independent analysis of both azacitidine and decitabine with respect to OS and time to AML/death revealed an advantage seen only with azacitidine (OS: azacitidine $[\mathrm{HR}=0.56 ; 95 \% \mathrm{CI}, 0.44-0.73]$ vs decitabine $[\mathrm{HR}=0.88$, 95\% CI, 0.66-1.17]) (time to AML/death: azacitidine $[\mathrm{HR}=0.54 ; 95 \% \mathrm{CI}, 0.42-0.71]$ vs decitabine $[\mathrm{HR}=0.85$; 95\% CI, 0.66-1.07). Although both drugs show encouraging clinical responses, the OS and delayed time to AML benefit seen only with azacitidine trials are interesting. The studies analyzed had similar proportion of IPSS risk groups/FAB

Table I Treatment approach in nontransplant candidates at the University of Minnesota

\begin{tabular}{ll}
\hline INT-I/low-risk & No treatment indicated $\rightarrow$ supportive measures only \\
IPSS & - Symptomatic anemia \\
If anemic + low EPO level: trial of EPO & supplementation with or without G-CSF \\
- Transfusion-dependent anemia not responding & to growth factors \\
& 5q-: lenalidomide \\
& Normal/other cytogenetics: order of treatment \\
choice & I) Azacitidine \\
& 2) Clinical trial \\
& 3) ATG/CSA: if younger (<60 years) or HLA DRI5 \\
& - Neutropenic or thrombocytopenic requiring therapy \\
& I) Azacitidine \\
& 2) Clinical trial if not responding to azacitidine \\
- Requiring therapy \\
INT-2/high-risk & Azacitidine \\
IPSS & Clinical trial if progression on azacitidine \\
\hline
\end{tabular}

Abbreviations: EPO, erythropoietin; G-CSF, granulocyte-colony-stimulating factor; ATG, antithymocyte globulin; CSA, cyclosporine; HLA-DRI5, human leukocyte antigen DR-15. 
(French American British) groups and similar median ages; however, the duration of decitabine therapy was slightly lower with a median of 3-4 cycles vs 9 cycles with azacitidine. ${ }^{35}$ The differing treatment exposure may explain the differing impact on OS benefit; however, only randomized studies comparing azacitidine and decitabine will answer the question of equivalence or superiority. Both agents produce desirable clinical responses and are reasonable therapeutic tools, but given the documented benefits of improved OS and delayed time to AML in the current literature, azacitidine has a mild advantage. Limited data does suggest clinical activity of decitabine in those patients with MDS whose disease stopped responding to azacitidine (ORR 28\%) possibly suggesting some additional mechanisms of action and the importance of both hypomethylating agents in MDS. ${ }^{54}$

\section{Current clinical investigations}

Numerous clinical trials evaluating novel therapeutic combinations for MDS are currently open and recruiting, although active studies are primarily limited to phase $1 / 2$ investigations. Of interest are several studies using azacitidine or decitabine in combination with various histone deacetylase inhibitors (vorinostat, panobinostat, and entinostat) potentially enhancing current epigenetic-based therapy. Azacitidine is also being studied in combination with agents, such as lenalidomide and bortezomib. Studies evaluating azacitidine in oral form, hypomethylating agents prior to allogeneic stem cell transplantation or in the role of maintenance therapy for MDS treated with intensive chemotherapy, are ongoing. A community-based, phase 4 trial of azacitidine vs decitabine has recently started recruiting. ${ }^{55}$

In small studies, a number of additional agents have documented clinical activity in MDS. Specifically, clofarabine, which has shown promise in older, newly diagnosed, and relapsed refractory patients with AML, has been tested in the oral form in patients with MDS yielding a $25 \%$ complete response rate. ${ }^{56-58}$ Combinations of antithymocyte globulin and etanercept have also shown evidence of HI with ORRs of approximately 56\% in small-scale studies. ${ }^{59}$ Studies involving bortezomib, arsenic trioxide, tipifarnib, a farnesyl-transferase inhibitor, and tyrosine kinase inhibitor, dasatinib, and many others are ongoing. ${ }^{55}$

\section{Conclusion}

Therapeutic options for the MDS have improved significantly over the last decade. However, given the variability in natural history of

Table 2 Treatment approach in transplant candidates at the University of Minnesota

\begin{tabular}{|c|c|}
\hline \multirow[t]{6}{*}{ Low-risk IPSS } & Supportive care as above \\
\hline & Due to potential long natural history, transplant typically not indicated up front, even in young patients, unless \\
\hline & showing evidence of progression \\
\hline & Note: Patients with transfusion dependency not responding to therapy (growth factors, hypomethylating agents, \\
\hline & etc) should be transplanted earlier despite their low-risk IPSS score due to higher TRM noted with higher \\
\hline & pretransplant ferritin levels and transfusion dependence \\
\hline \multirow[t]{15}{*}{ INT-I IPSS } & Age $\geq 50$ years: Poorer outcomes compared with younger patients. Thus, consideration of transplantation at diagnosis \\
\hline & If $<5 \%$ blasts in marrow at diagnosis: Transplant with no up front therapy \\
\hline & If $>5 \%$ blasts in marrow at diagnosis: Initiate therapy to reduce blast percentage and proceed to transplant \\
\hline & Therapy choice \\
\hline & Azacitidine: Ist choice if have time to achieve benefit \\
\hline & AML type induction chemotherapy: If high proliferative capacity and need urgent response \\
\hline & If age $<50$ years: May have prolonged natural history without significant intervention, so no transplant up front unless \\
\hline & failure of below interventions or progressive cytopenias/disease progression \\
\hline & Supportive care if minimal cytopenias \\
\hline & Thrombocytopenic/neutropenic requiring treatment: \\
\hline & Azacitidine \\
\hline & Symptomatic anemia requiring transfusions \\
\hline & 5q-: patients: Lenalidomide \\
\hline & Normal/other cytogenetics: Azacitidine \\
\hline & If progressed through above therapies and need further disease reduction prior to transplantation $\rightarrow$ clinical trial \\
\hline INT-2/high-risk & All age patients $(<70-75$ years $)$ \\
\hline \multirow[t]{5}{*}{ IPSS/t-MDS } & Prepare for allogeneic HCT from diagnosis: HLA type patient, sibs, initiate MUD/cord search if no sibling match \\
\hline & Treatment, as needed, to reach goal of $<5 \%$ blasts \\
\hline & Azacitidine Ist choice: (needs 3 to 4 cycles to assess true response) \\
\hline & Induction chemotherapy if high proliferative rate \\
\hline & Clinical trial if suboptimal response to above and need further disease reduction pretransplant \\
\hline
\end{tabular}

Abbreviations: TRM, treatment-related mortality; AML, acute myeloid leukemia; t-MDS, treatment-related MDS; HCT, hematopoietic stem cell transplant; HLA, human leukocyte antigen; MUD, matched unrelated donor. 
disease, the variety of treatment options, and the potential morbidity and mortality of specific therapies, MDS treatment decisionmaking is challenging with respect to timing of therapy initiation, therapeutic agent choice, and timing of transplant, if planned. Our treatment approach at the University of Minnesota has based decision-making on diagnostic IPSS score, WPSS (WHO-based Prognostic Scoring System) score, cytogenetics, lineages impacted, and transplant candidacy and is summarized in Tables 1 and 2.

Hypomethylating agents (azacitidine and decitabine) are integral to the current management of patients with MDS. ${ }^{35,60,61}$ When using these agents, it is important to remember that a minimum of 4 cycles should be given, in the absence of toxicity or rapidly progressive disease, to allow for adequate chance for response. Once a response is achieved, therapy should be continued until disease progression or treatment-related toxicity precludes further therapy. Hypomethylating agents may be effective therapy in additional roles, such as maintenance therapy after induction chemotherapy or up front therapy for elderly patients with AML not proceeding to transplant, maintenance therapy after allogeneic stem cell transplantation for MDS attempting to reduce risk of relapse, or combined with other agents for patients with MDS unresponsive to standard therapy. Clinical trials are underway assessing the efficacy of azacitidine and decitabine within these clinical contexts.

Continued research is crucial to develop new therapies for patients with MDS with the hopes of providing curative therapeutic alternatives to stem cell transplantation.

\section{Disclosures}

The authors report no conflicts of interest in this work.

\section{References}

1. Warlick ED, Smith BD. Myelodysplastic syndromes: review of pathophysiology and current novel treatment approaches. Curr Cancer Drug Targets. 2007;7(6):541-558.

2. Herman JG, Civin CI, Issa JP, et al. Distinct patterns of inactivation of p15INK4B and p16INK4A characterize the major types of hematological malignancies. Cancer Res. 1997;57(5):837-841.

3. Leone G, Teofili L, Voso MT, Lubbert M. DNA methylation and demethylating drugs in myelodysplastic syndromes and secondary leukemias. Haematologica. 2002;87(12):1324-1341.

4. Quesnel B, Guillerm G, Vereecque R, et al. Methylation of the p15(INK4b) gene in myelodysplastic syndromes is frequent and acquired during disease progression. Blood. 1998;91(8):2985-2990.

5. Aggerholm A, Holm MS, Guldberg P, Olesen LH, Hokland P. Promoter hypermethylation of p15INK4B, HIC1, CDH1, and ER is frequent in myelodysplastic syndrome and predicts poor prognosis in early-stage patients. Eur J Haematol. 2006;76(1):23-32.

6. Christiansen DH, Andersen MK, Pedersen-Bjergaard J. Methylation of p15INK4B is common, is associated with deletion of genes on chromosome arm $7 \mathrm{q}$ and predicts a poor prognosis in therapy-related myelodysplasia and acute myeloid leukemia. Leukemia. 2003;17(9): 1813-1819.
7. Kantarjian H, Issa JP, Rosenfeld CS, et al. Decitabine improves patient outcomes in myelodysplastic syndromes: results of a phase III randomized study. Cancer. 2006;106(8):1794-1803.

8. Kantarjian H, Oki Y, Garcia-Manero G, et al. Results of a randomized study of 3 schedules of low-dose decitabine in higher-risk myelodysplastic syndrome and chronic myelomonocytic leukemia. Blood. 2007;109(1):52-57.

9. Silverman LR, Demakos EP, Peterson BL, et al. Randomized controlled trial of azacitidine in patients with the myelodysplastic syndrome: a study of the Cancer and Leukemia Group B. J Clin Oncol. 2002;20(10):2429-2440.

10. Silverman LR, McKenzie DR, Peterson BL, et al. Further analysis of trials with azacitidine in patients with myelodysplastic syndrome: studies 8421, 8921, and 9221 by the Cancer and Leukemia Group B. J Clin Oncol. 2006;24(24):3895-3903.

11. Fenaux P, Mufti GJ, Hellstrom-Lindberg E, et al. Efficacy of azacitidine compared with that of conventional care regimens in the treatment of higher-risk myelodysplastic syndromes: a randomised, open-label, phase III study. Lancet Oncol. 2009;10(3):223-232.

12. Kaminskas E, Farrell A, Abraham S, et al. Approval summary: azacitidine for treatment of myelodysplastic syndrome subtypes. Clin Cancer Res. 2005;11(10):3604-3608.

13. Stresemann C, Lyko F. Modes of action of the DNA methyltransferase inhibitors azacytidine and decitabine. Int J Cancer. 2008; 123(1):8-13.

14. Issa JP, Kantarjian HM. Targeting DNA methylation. Clin Cancer Res. 2009;15(12):3938-3946.

15. Griffiths EA, Gore SD. DNA methyltransferase and histone deacetylase inhibitors in the treatment of myelodysplastic syndromes. Semin Hematol. 2008;45(1):23-30.

16. Fandy TE, Carraway H, Gore SD. DNA demethylating agents and histone deacetylase inhibitors in hematologic malignancies. Cancer $J$. 2007;13(1):40-48.

17. Schermelleh L, Spada F, Easwaran HP, et al. Trapped in action: direct visualization of DNA methyltransferase activity in living cells. Nat Methods. 2005;2(10):751-756.

18. Fandy TE, Herman JG, Kerns P, et al. Early epigenetic changes and DNA damage do not predict clinical response in an overlapping schedule of 5-azacytidine and entinostat in patients with myeloid malignancies. Blood. 2009;114(13):2764-2773.

19. Gore SD. In vitro basis for treatment with hypomethylating agents and histone deacetylase inhibitors: can epigenetic changes be used to monitor treatment? Leuk Res. 2009;33 Suppl 2:S2-S6.

20. Shen L, Kantarjian H, Guo Y, et al. DNA methylation predicts survival and response to therapy in patients with myelodysplastic syndromes. J Clin Oncol. 2010;28(4):605-613.

21. Karon M, Sieger L, Leimbrock S, et al. 5-Azacytidine: a new active agent for the treatment of acute leukemia. Blood. 1973;42(3):359-365.

22. McCredie KB, Bodey GP, Burgess MA, et al. Treatment of acute leukemia with 5-azacytidine (NSC-102816). Cancer Chemother Rep. 1973;57(3):319-323.

23. Shnider BI, Baig M, Colsky J. A phase I study of 5-azacytidine (NSC102816). J Clin Pharmacol. 1976;16(4):205-212.

24. Vogler WR, Miller DS, Keller JW. 5-Azacytidine (NSC 102816): a new drug for the treatment of myeloblastic leukemia. Blood. 1976; 48(3):331-337.

25. US Food and Drug Administration. http://www.accessdata.fda.gov/ drugsatfda_docs/label/2008/050794s011lbl.pdf. Accessed Feb 1, 2010.

26. Lyons RM, Cosgriff TM, Modi SS, et al. Hematologic response to three alternative dosing schedules of azacitidine in patients with myelodysplastic syndromes. J Clin Oncol. 2009;27(11):1850-1856.

27. MacBeth KJ, Laille E, Ning Y, et al. A comparative pharmacokinetic/ pharmacodynamic (PK/PD) evaluation of azacitidine following subcutaneous (SC) and oral administration in subjects with myelodysplastic syndromes (MDS) or acute myelogenous leukemia (AML), results from a phase 1 study. ASH Annu Meet Abstr. 2009;114(22):1772. 
28. Garcia-Manero G, Gore SD, Skikne B, et al. A phase 1, open-label, dose-escalation study to evaluate the safety, pharmacokinetics, and pharmacodynamics of oral azacitidine in patients with myelodysplastic syndromes (MDS) or acute myelogenous leukemia (AML). ASH Annu Meet Abstr. 2009;114(22):117.

29. Troetel WM, Weiss AJ, Stambaugh JE, Laucius JF, Manthei RW. Absorption, distribution, and excretion of 5-azacytidine (NSC-102816) in man. Cancer Chemother Rep. 1972;56(3):405-411.

30. Kaminskas E, Farrell AT, Wang YC, Sridhara R, Pazdur R. FDA drug approval summary: azacitidine (5-azacytidine, Vidaza) for injectable suspension. Oncologist. 2005;10(3):176-182.

31. Chabot GG, Rivard GE, Momparler RL. Plasma and cerebrospinal fluid pharmacokinetics of 5-Aza-2'-deoxycytidine in rabbits and dogs. Cancer Res. 1983;43(2):592-597.

32. Silverman LR, Holland JF, Weinberg RS, et al. Effects of treatment with 5-azacytidine on the in vivo and in vitro hematopoiesis in patients with myelodysplastic syndromes. Leukemia. 1993;7 Suppl 1: S21-S29.

33. Cheson BD, Bennett JM, Kantarjian H, et al. Report of an international working group to standardize response criteria for myelodysplastic syndromes. Blood. 2000;96(12):3671-3674.

34. Kornblith AB, Herndon JE II, Silverman LR, et al. Impact of azacytidine on the quality of life of patients with myelodysplastic syndrome treated in a randomized phase III trial: a Cancer and Leukemia Group B study. J Clin Oncol. 2002;20(10):2441-2452.

35. Gurion R, Vidal L, Gafter-Gvili A, et al. 5-azacitidine prolongs overall survival in patients with myelodysplastic syndrome - a systematic review and meta-analysis. Haematologica. 2010;95(2):303-310.

36. Maslak P, Chanel S, Camacho LH, et al. Pilot study of combination transcriptional modulation therapy with sodium phenylbutyrate and 5-azacytidine in patients with acute myeloid leukemia or myelodysplastic syndrome. Leukemia. 2006;20(2):212-217.

37. Braiteh F, Soriano AO, Garcia-Manero G, et al. Phase I study of epigenetic modulation with 5-azacytidine and valproic acid in patients with advanced cancers. Clin Cancer Res. 2008;14(19):6296-6301.

38. Soriano AO, Yang H, Faderl S, et al. Safety and clinical activity of the combination of 5-azacytidine, valproic acid, and all-trans retinoic acid in acute myeloid leukemia and myelodysplastic syndrome. Blood. 2007;110(7):2302-2308.

39. Nand S, Godwin J, Smith S, et al. Hydroxyurea, azacitidine and gemtuzumab ozogamicin therapy in patients with previously untreated non-M3 acute myeloid leukemia and high-risk myelodysplastic syndromes in the elderly: results from a pilot trial. Leuk Lymphoma. 2008;49(11):2141-2147.

40. Kenealy MK, Seymour JF, Linda C, et al. The tolerability of combination therapy with thalidomide and 5-azacitidine in patients with advanced myelodysplastic syndromes (MDS). ASH Annu Meet Abstr. 2009;114(22):1749.

41. Sekeres MA, List AF, Cuthbertson D, et al. Phase I combination trial of lenalidomide and azacitidine in patients with higher-risk myelodysplastic syndromes. J Clin Oncol. 2010;28(13):2253-2258.

42. De Padua Silva L, de Lima M, Kantarjian H, et al. Feasibility of alloSCT after hypomethylating therapy with decitabine for myelodysplastic syndrome. Bone Marrow Transplant. 2009;43(11):839-843.

43. Field T, Perkins J, Huang Y, et al. 5-Azacitidine for myelodysplasia before allogeneic hematopoietic cell transplantation. Bone Marrow Transplant. 2010;45(2):255-260.

OncoTargets and Therapy

\section{Publish your work in this journal}

OncoTargets and Therapy is an international, peer-reviewed, open access journal focusing on the pathological basis of all cancers, potential targets for therapy and treatment protocols employed to improve the management of cancer patients. The journal also focuses on the impact of management programs and new therapeutic agents and protocols on
44. Sanchez-Abarca LI, Gutierrez-Cosio S, Santamaria C, et al. Immunomodulatory effect of 5-azacytidine (5-azaC): potential role in the transplantation setting. Blood. 2010;115(1):107-121.

45. Platzbecker U, Aul C, Ehninger G, Giagounidis A. Reduction of 5 -azacitidine induced skin reactions in MDS patients with evening primrose oil. Ann Hematol. 2010;89(4):427-428.

46. Pinchon DJ, Stanworth SJ, Doree C, Brunskill S, Norfolk DR. Quality of life and use of red cell transfusion in patients with myelodysplastic syndromes. A systematic review. Am J Hematol. 2009;84(10):671-677.

47. Caocci G, Baccoli R, Ledda A, Littera R, La Nasa G. A mathematical model for the evaluation of amplitude of hemoglobin fluctuations in elderly anemic patients affected by myelodysplastic syndromes: correlation with quality of life and fatigue. Leuk Res. 2007;31(2):249-252.

48. Oliva EN, Dimitrov BD, Benedetto F, D’Angelo A, Nobile F. Hemoglobin level threshold for cardiac remodeling and quality of life in myelodysplastic syndrome. Leuk Res. 2005;29(10):1217-1219.

49. Stasi R, Abruzzese E, Lanzetta G, Terzoli E, Amadori S. Darbepoetin alfa for the treatment of anemic patients with low- and intermediate-1-risk myelodysplastic syndromes. Ann Oncol. 2005;16(12):1921-1927.

50. Balleari E, Rossi E, Clavio M, et al. Erythropoietin plus granulocyte colony-stimulating factor is better than erythropoietin alone to treat anemia in low-risk myelodysplastic syndromes: results from a randomized single-centre study. Ann Hematol. 2006;85(3):174-180.

51. Casadevall N, Durieux P, Dubois S, et al. Health, economic, and qualityof-life effects of erythropoietin and granulocyte colony-stimulating factor for the treatment of myelodysplastic syndromes: a randomized, controlled trial. Blood. 2004;104(2):321-327.

52. Gabrilove J, Paquette R, Lyons RM, et al. Phase 2, single-arm trial to evaluate the effectiveness of darbepoetin alfa for correcting anaemia in patients with myelodysplastic syndromes. Br J Haematol. 2008; 142(3):379-393.

53. Steensma DP, Heptinstall KV, Johnson VM, et al. Common troublesome symptoms and their impact on quality of life in patients with myelodysplastic syndromes (MDS): results of a large internet-based survey. Leuk Res. 2008;32(5):691-698.

54. Borthakur G, Ahdab SE, Ravandi F, et al. Activity of decitabine in patients with myelodysplastic syndrome previously treated with azacitidine. Leuk Lymphoma. 2008;49(4):690-695.

55. ClinicalTrials.gov. Available from: http://www.clinicaltrials.gov. Accessed May 9, 2010.

56. Faderl S, Verstovsek S, Cortes J, et al. Clofarabine and cytarabine combination as induction therapy for acute myeloid leukemia (AML) in patients 50 years of age or older. Blood. 2006;108(1):45-51.

57. Faderl S, Gandhi V, O'Brien S, et al. Results of a phase 1-2 study of clofarabine in combination with cytarabine (ara-C) in relapsed and refractory acute leukemias. Blood. 2005;105(3):940-947.

58. Faderl S, Garcia-Manero G, Estrov Z, et al. Oral clofarabine in the treatment of patients with higher-risk myelodysplastic syndrome. J Clin Oncol. 2010;28(16):2755-2760.

59. Scott BL, Ramakrishnan A, Fosdal M, et al. Anti-thymocyte globulin plus etanercept as therapy for myelodysplastic syndromes (MDS): a phase II study. Br J Haematol. 2010;149(5):706-710.

60. Musolino C, Sant'antonio E, Penna G, et al. Epigenetic therapy in myelodysplastic syndromes. Eur J Haematol. 2010;84(6):463-467.

61. Fenaux P, Ades L. Review of azacitidine trials in intermediate-2-and high-risk myelodysplastic syndromes. Leuk Res. 2009;33 Suppl 2: S7-S11.

\section{Dovepress}

patient perspectives such as quality of life, adherence and satisfaction. The manuscript management system is completely online and includes a very quick and fair peer-review system, which is all easy to use. Visit http://www.dovepress.com/testimonials.php to read real quotes from published authors. 